\title{
Inhibition of EMMPRIN and MMP-9 Expression by Epigallocatechin-3-Gallate through 67-kDa Laminin Receptor in PMA- Induced Macrophages
}

\author{
Qi-Ming Wang Hao Wang Ya-Fei Li Zhi-Yong Xie Yao Ma Jian-Jun Yan Yi Fan \\ Wei Gao Ze-Mu Wang Lian-Sheng Wang
}

Department of Cardiology, the First Affiliated Hospital of Nanjing Medical University, Nanjing, China

\section{Key Words}

Epigallocatechin-3-gallate - 67-kDa laminin receptor - MMP-9 - EMMPRIN • Stabilization of atherosclerotic plaque

\begin{abstract}
Background/Aims: It is well documented that overexpression of EMMPRIN (extracellular matrix metalloproteinase inducer) and MMPs (matrix metalloproteinases) by monocytes/ macrophages plays an important role in atherosclerotic plaque rupture. Green tea polyphenol epigallocatechin-3-gallate (EGCG) has a variety of pharmacological properties and exerts cardiovascular protective effects. Recently, the 67-kD laminin receptor (67LR) has been identified as a cell surface receptor of EGCG. The aim of the present study was to evaluate the effects of EGCG on the expression of EMMPRIN and MMP-9 in PMA-induced macrophages, and the potential mechanisms underlying its effects. Methods: Human monocytic THP-1 cells were induced to differentiate into macrophages with phorbol 12-myristate 13-acetate (PMA). Protein expression and MMP-9 activity were assayed by Western blot and Gelatin zymography, respectively. Real-time PCR was used to examine EMMPRIN and MMP-9 mRNA expression. Results: We showed that EGCG $(10-50 \mu \mathrm{mol} / \mathrm{L})$ significantly inhibited the expression of EMMPRIN and MMP-9 and activation of extracellular signal-regulated kinase 1/2 (ERK1/2), p38 and c-Jun N-terminal kinase (JNK) in PMA-induced macrophages. Downregulation of EMMPRIN by gene silencing hindered PMA-induced MMP-9 secretion and expression, indicating an important role of EMMPRIN in the inhibition of MMP-9 by EGCG. Moreover, 67LR was involved in EGCG-mediated suppression of EMMPRIN and MMP-9 expression. Anti-67LR antibody treatment led to abrogation of the inhibitory action of EGCG on the expression of EMMPRIN and MMP-9 and activation of ERK1/2, p38, and JNK. Conclusion: Our results indicate that EGCG restrains EMMPRIN and MMP-9 expression via 67LR in PMAinduced macrophages, which also suggests that EGCG may be a possible therapeutic agent for stabilizing atherosclerotic plaque.




\section{Cellular Physiology Cell Physiol Biochem 2016;39:2308-2319

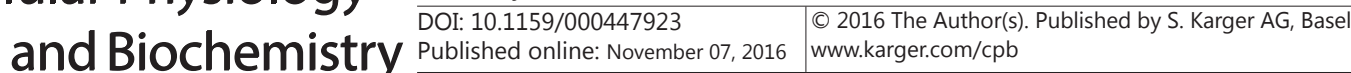 \\ Wang et al.: Effects of EGCG on the Expression of EMMPRIN and MMP-9}

\section{Introduction}

Acute coronary syndrome (ACS) refers to a range of acute myocardial ischaemic states, which includes unstable angina and acute myocardial infarction (AMI) with or without ST elevation. Most of the acute coronary syndromes are considered to result from plaque rupture and coronary thrombosis [1]. Numerous reports have shown that this rupture depends on the destruction of the extracellular matrix (ECM) by MMPs [2]. MMP-9, one of the most prevalent MMPs produced by monocytes/macrophages, is highly expressed in the vulnerable regions of advanced atherosclerotic plaque, and it has been suggested it is associated with plaque progression and destabilization [3-6].

Basigin (CD147), a member of the immunoglobulin superfamily, was initially found on the membranes of tumor cells [7]. CD147 was shown to have the ability to induce the synthesis of MMPs, and was renamed EMMPRIN (extracellular matrix metalloproteinase inducer) $[8,9]$. EMMPRIN was found to stimulate MMP-9 expression in monocytes and MMP2 activity in human smooth muscle cells $[5,10]$. Furthermore, overexpression of EMMPRIN was observed in inflammatory disease such as lung inflammatory disease, rheumatoid arthritis and chronic liver disease [11-13]. In recent years, a significant amount of evidence has documented the pivotal role of EMMPRIN in the complex processes of atherosclerosis and acute atherosclerothrombosis [14]. EMMPRIN was reported overexpressed in human atherosclerotic plaques, and was colocalized with macrophages/monocytes [15]. In addition, CD147 expression on the surface of circulating monocytes was significantly upregulated in patients with acute coronary syndrome $[5,16]$.

Green tea is a commonly consumed beverage throughout the world. Regular consumption of green tea is associated with reduced risk of cardiovascular diseases and cancer $[17,18]$. Green tea leaves are a rich source of extractable polyphenols, commonly known as catechins. These catechins are mainly comprised of (-)-epigallocatechin-3-gallate (EGCG), (-)-epicatechin (EC), (-)-epigallocatechin (EGC), and (-)-epicatechin-3-gallate (ECG). EGCG, the most abundant and active compound, exhibits a wide range of biological and pharmacological properties, such as anticancer, antioxidant, anti-inflammatory and antiatherosclerotic activities $[17,18]$. Recently, the $67-\mathrm{kDa}$ laminin receptor $(67 \mathrm{LR})$, a nonintegrin-type cell surface-associated receptor, has been identified as a cell surface receptor of EGCG [19] and plays a central role in the anticancer [20,21] and anti-inflammatory effects of EGCG [22-24]. We also found that EGCG inhibited tumor necrosis factor- $\alpha$ (TNF- $\alpha$ )-induced monocyte chemo attractant protein-1 (MCP-1) expression in HUVECs through 67LR [25].

Some studies have shown the antiatherosclerotic activity of EGCG. However, the effect of EGCG on atherosclerotic plaque stability is unclear. EMMPRIN and MMP-9 expressed by monocytes/macrophages can promote instability of atherosclerotic plaque, inhibition of EMMPRIN and MMP-9 expression is therefore interesting for the aim of stabilization of atherosclerotic plaque. In the present study, we aimed to elucidate the effects and underlying molecular mechanisms of EGCG on the expression of MMP-9 and EMMPRIN in PMA-induced macrophages. Moreover, we assessed the involvement of 67LR in the downregulation of EMMPRIN and MMP-9 by EGCG.

\section{Materials and Methods}

\section{Cell Culture and Treatment}

Human monocytic cell line THP-1 was obtained from American Type Culture Collection (ATCC, Manassas, VA, USA). The cells were maintained at a density of $1 \times 10^{6} / \mathrm{ml}$ in RPMI 1640 medium (Life Technologies, Carlsbad, CA, USA) containing 10\% FBS (Hyclone, Logan, Utah, USA) and 1\% pen/strep solution at $37^{\circ} \mathrm{C}$ in a $5 \% \mathrm{CO} 2$ incubator. To induce macrophage differentiation, THP-1 cells were cultured in six-well plates in the presence of 100 nM PMA (Sigma, Louis, MO, USA) for 48 hours [26]. EGCG, PD98059, SB203580, and SP600125 were purchased from Sigma Company (Louis, MO, USA). The cells were pretreated with EGCG (10, 25, and $50 \mu \mathrm{M})$, PD98059, SB203580, and SP600125 for 1 hour, then cultured with PMA for 


\section{Cellular Physiology Cell Physiol Biochem 2016;39:2308-2319

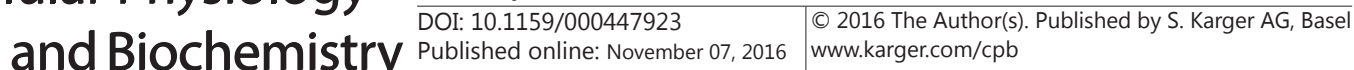 \\ Wang et al.: Effects of EGCG on the Expression of EMMPRIN and MMP-9}

another 48 hours. For the blockage of 67LR, cells were pre-incubated with 67LR-blocking antibody (clone MluC5; $20 \mu \mathrm{g} / \mathrm{ml}$; Thermo Fisher Scientific, Waltham, MA, USA) or control mouse IgM (20 $\mu \mathrm{g} / \mathrm{ml})$ for 1 hour before the treatment of EGCG.

\section{Determination of cell viability}

The cytotoxicity of EGCG on PMA-induced macrophages was examined using the Cell Counting Kit-8 Assay Kit (Dojindo Laboratories, Kumamoto, Japan). PMA-induced macrophages were seeded in 96-well plates at $1 \times 10^{4}$ cells/well. Twenty-four hours later, the cells were incubated with EGCG at increasing concentrations ( 0 to $100 \mathrm{uM}$ ) for 48 hours. After $10 \mu \mathrm{l} \mathrm{CCK-8} \mathrm{solution} \mathrm{was} \mathrm{added} \mathrm{to} \mathrm{each} \mathrm{well} \mathrm{and} \mathrm{incubated}$ for 4 hours, the absorbance at $450 \mathrm{~nm}$ was measured using Synergy 2 microplate reader (BioTek, Winooski, VT, USA). The viability of PMA-induced macrophages was determined according to the manufacturer's instructions.

RNA isolation, cDNA synthesis and real-time PCR

Total cellular RNA was isolated from PMA-induced macrophages using Trizol reagent (Invitrogen, Carlsbad, CA, USA) according to the manufacturer's instructions and reverse-transcribed into cDNA using the Reverse Transcription Kit (Takara, Dalian, China). The real-time polymerase chain reaction (PCR) was carried out using the ABI 7900 Real Time PCR apparatus with Power SYBR green PCR Master Mix (Applied Biosystems, Foster, CA, USA). The primer sequences are shown in Table 1. The conditions for quantitative real-time PCR were: $95^{\circ} \mathrm{C}$ for 10 minutes, then 40 cycles at $95^{\circ} \mathrm{C}$ for 15 seconds, and $60^{\circ} \mathrm{C}$ for 1 minute, and a final extension of $95^{\circ} \mathrm{C}$ for 15 seconds, $60^{\circ} \mathrm{C}$ for 1 minute and $95^{\circ} \mathrm{C}$ for 15 seconds. The expression levels of target genes were normalized against the GAPDH level.

Small-interfering RNA-mediated gene silencing of EMMPRIN

Small-interfering RNA (siRNA) for EMMPRIN [sense, 5'-GCACAGUCUUCACUACCGUTT-3'; antisense, 5'-ACGGUAGUGAAGACUGUGCTT-3'] and scrambled control siRNA were designed by Invitrogen (Carlsbad, CA, USA). The siRNA were transfected into THP-1 cells by using the Lipofectamine-3000 (Invitrogen, Carlsbad, CA, USA). The cells were incubated with the siRNA for 48 hours prior to treatment with PMA and were then analyzed by Western Blot.

\section{Protein isolation and Western blot analysis}

Treated THP-1 macrophages were harvested and lysed. Cell lysates were incubated on ice for 30 minutes followed by centrifugation for 20 minutes at $12,000 \mathrm{rpm}, 4^{\circ} \mathrm{C}$. The protein concentration of the cell extracts was measured using the BCA Protein Kit Assay (Takara, Dalian, China). Protein extracts were electrophoresed through $12 \%$ polyacrylamide gels and transferred onto polyvinylidene difluoride (PVDF) membranes. The membranes then were blocked with 5\% BSA in TBST buffer (10 mM Tris, pH 7.5, $150 \mathrm{mM} \mathrm{NaCl}$, and $0.05 \%$ Tween-20) for 2 hours at room temperature followed by incubation overnight at $4{ }^{\circ} \mathrm{C}$ with primary antibodies for EMMPRIN (Abcam, Cambridge, UK), MMP-9, phospho-ERK1/2, ERK1/2, phospho-p38, p38, phospho-JNK, JNK, GAPDH (Cell Signaling Technology, Boston, MA, USA) and 67LR (Thermo Fisher Scientific, Waltham, MA, USA). After washing 3 times for 10 minutes each in TBST, the membranes were incubated with horseradish peroxidase-conjugated anti-rabbit (Biosynthesis, Beijing, China) or anti-mouse (Santa Cruz, Dallas, TX, USA) secondary antibodies at room temperature for 1 hour. Lastly, membranes were washed 3 times for 10 minutes each in TBST and developed with enhanced chemiluminescence. The immunoreactivity was detected using ChemiDoc XRS Imaging System (BioRad, Hercules, CA, USA).

Table 1. Primers of EMMPRIN, MMP-9 and GAPDH for Real-time PCR

\begin{tabular}{lll}
\hline Target & Primers & \\
\hline \multirow{2}{*}{ EMMPRIN } & Sense: & 5'-AAGTCGTCAGAACACATCAACG-3' \\
& Antisense: & 5'-AACTCACGAAGAACCTGCTCTC-3 \\
& Sense: & 5'-TTGACAGCGACAAGAAGTGG-3' \\
MMP-9 & Antisense: & 5'-CCCTCAGTGAAGCGGTACAT-3' \\
& Sense: & 5'-GGTGGTCTCCTCTGACTTCAACA-3' \\
GAPDH & Antisense: & 5'-GTTCTCGTAGCCAAATTCGTTGT-3' \\
& &
\end{tabular}

\section{Gelatin zymography}

THP-1 cells were seeded at a density of $3 \times 10^{5}$ cells per well in 6 -well plate. Cells were incubated in serum-free medium with or without 


\section{Cellular Physiology Cell Physiol Biochem 2016;39:2308-2319

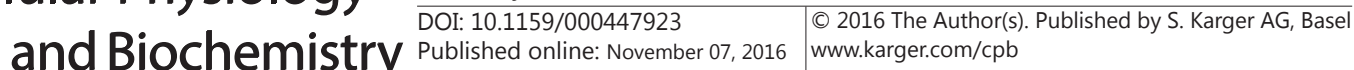 \\ Wang et al.: Effects of EGCG on the Expression of EMMPRIN and MMP-9}

EGCG (10, 25 and $50 \mu \mathrm{M})$ for 1 hour, and then cultured with PMA for another 48 hours. Culture supernatants were collected, and $10 \mu \mathrm{l}$ aliquots of the culture supernatant were electrophoresed through $10 \%$ polyacrylamide gels containing $1 \mathrm{mg} / \mathrm{ml}$ gelatin. After electrophoresis, the gels were washed twice for 15 minutes each with $2.5 \%$ Triton $\mathrm{X}-100$ at $37^{\circ} \mathrm{C}$ and were then immersed in developing buffer (10 mM Tris Base, $40 \mathrm{mMT}$ ris- $\mathrm{HCl}, 200 \mathrm{mMNaCl}, 10 \mathrm{mMCaCl} 2,0.02 \%$ Brij 35) for 11 hours at $37^{\circ} \mathrm{C}$. Subsequently, the gels were stained for 2 hours with $0.5 \%$ Coomassie Blue R-250 followed by treatment with destaining buffer (50\% methanol, $10 \%$ glacial acetic acid, 40\% water). A clear white band showing MMP-9 activity was detected against a blue background. The gels were photographed by ChemiDoc XRS Imaging System (Bio-Rad, Hercules, CA, USA).

\section{Statistical analysis}

The data were analyzed using SPSS statistics software, version 17.0 (SPSS Inc., Chicago, IL, USA). Data were expressed as mean \pm standard deviation (SD). Statistical significance was assessed by one-way ANOVA or Student's t-test. It was considered statistically significant when the P-value was less than 0.05.

\section{Results}

\section{Cell differentiation and cytotoxicity of EGCG}

THP-1 cells grew as a single-cell suspension. After treatment with $100 \mathrm{nM}$ Phorbol 12-myristate 13-acetate (PMA) for 48 hours, THP-1 cells were differentiated into adherent macrophages (Fig. 1a, b). The structure of EGCG used in this study is shown in Fig. 1c. We performed a CCK-8 assay to assess the cytotoxicity of EGCG on PMA-induced macrophages. The cells were treated with a concentration of EGCG ranging from 0 to $100 \mu \mathrm{M}$ for 48 hours. As shown in Fig. 1d, EGCG up to $50 \mu \mathrm{M}$ did not lead to significant changes in cell viability. Therefore, we chose the EGCG dose less than $50 \mu \mathrm{M}$ for this experiment.

\section{Effect of EGCG on MMP-9 expression and activity}

Real-time PCR and Western blot were used to determine the expression of MMP9 mRNA and protein, respectively. As shown in Fig. 2a and 2d, MMP-9 mRNA and protein expression were markedly elevated after the exposure of THP-1 cells to $100 \mathrm{nM}$ PMA for 48 hours. However, pretreatment of the cells with the indicated concentrations of EGCG for 1 hour prior to PMA stimulation significantly inhibited MMP-9 mRNA and protein production in a concentration-dependent manner. We further measured gelatinolytic activities of MMP9 by SDS-polyacrylamide gelatin zymography assay. As shown in Fig. $2 b$ and 2d, MMP-9 enzymatic activity was also inhibited by EGCG in a concentration- dependent manner.

Fig. 1. Cell differentiation and cytotoxicity of EGCG. (a-b) Phase contrast images of Human monocytic cell line THP-1 and macrophages. (c) Structure of green tea epigallocatechin-3-gallate (EGCG). (d) Effects of EGCG on cell viability. PMA-induced macrophages were treated with EGCG at increasing concentrations $(0-100 \mu \mathrm{M})$ for 48 hours. CCK-8 assay was used to assess cell viability. Cells incubated in a medium without EGCG were defined as a control and considered $100 \%$ viable.

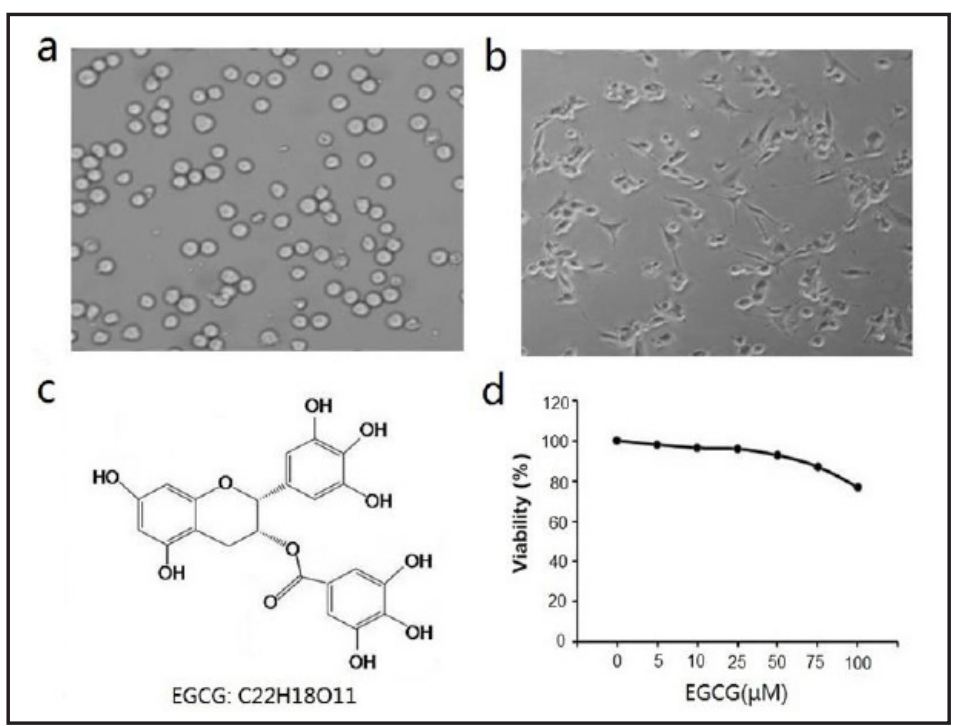




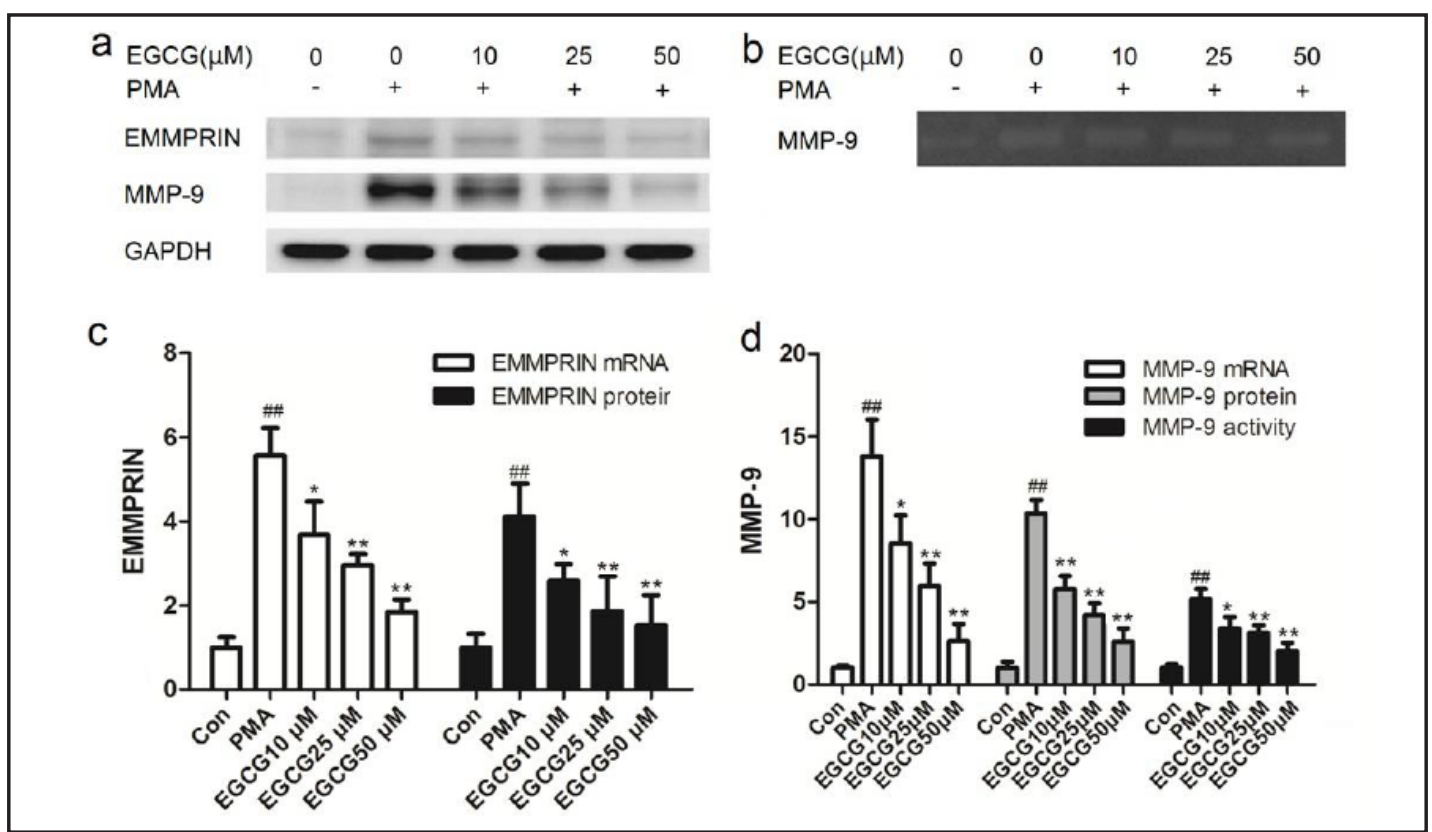

Fig. 2. Effects of EGCG on EMMPRIN and MMP-9 expression. (a) Representative Western blots for EMMPRIN and MMP-9 detection. (b) Representative gelatin zymography showing MMP-9 activity. (c) Real-time PCR results of EMMPRIN mRNA and densitometric analysis of EMMPRIN protein. (d) Real-time PCR results of MMP-9 mRNA and densitometric analysis of MMP-9 protein and activity. Data represent mean \pm S.D. of at least three independent experiments. \#\#: $\mathrm{P}<0.01$ vs control (con) group, *: $\mathrm{P}<0.05$ vs PMA group, ${ }^{* *}$ : $\mathrm{P}<$ 0.01 vs PMA group. Con indicates that THP-1 cells were cultured in a medium without EGCG and PMA. EGCG $(10,25$ and $50 \mu \mathrm{M})$ indicates that THP-1 cells were pre-incubated with different concentrations of EGCG before exposure to $100 \mathrm{nM}$ PMA for 48 hours.

\section{Effect of EGCG on EMMPRIN expression}

Considering EMMPRIN can induce the synthesis of MMP-9 and plays an important role in plaque rupture, we also investigated the effect of EGCG on EMMPRIN expression. The expression of EMMPRIN mRNA and protein were assayed by Real-time PCR and Western blot, respectively. Our results showed that EGCG significantly suppressed the expression of EMMPRIN mRNA and protein in a dose-dependent manner (Fig. 2a, c), which were similar to the inhibitory effect of EGCG on MMP-9 mRNA and protein expression.

\section{Involvement of CD147 in EGCG-mediated inhibition of MMP-9 expression}

We further investigated the relevance of EMMPRIN within the inhibitory action of EGCG on MMP-9 expression and activity. EMMPRIN gene silencing was performed using siRNA as described in Methods. As shown in Fig. 3a and 3c, EMMPRIN expression was lowered in PMAinduced macrophages transfected with siRNA, and EMMPRIN gene silencing suppressed EMMPRIN expression to a similar extent with EGCG $(50 \mu \mathrm{M})$. Furthermore, downregulation of EMMPRIN by gene silencing significantly reduced MMP-9 expression and enzymatic activity in PMA-induced macrophages. MMP-9 expression and activity were inhibited by $50.3 \%( \pm$ $14.0 \%$ ) and $41.5 \%( \pm 12.3 \%$ ), respectively (Fig. 3b, d). Taken together, these results indicate that the reduction of MMP-9 expression and activity is primary due to the down-regulation of EMMPRIN by EGCG.

\section{Effect of EGCG on MAPK signaling pathways}

Previous studies have shown that MAPK signaling pathways (ERK1/2, p38, and JNK) are stimulated by PMA $[27,28]$. In order to determine whether these kinases were involved in the up-regulation of EMMPRIN and MMP-9 expression, we examined the effect of PD98059 (PD, ERK1/2-specific inhibitor), SB203580 (SB, p38-specific inhibitor), and SP600125 (SP, 


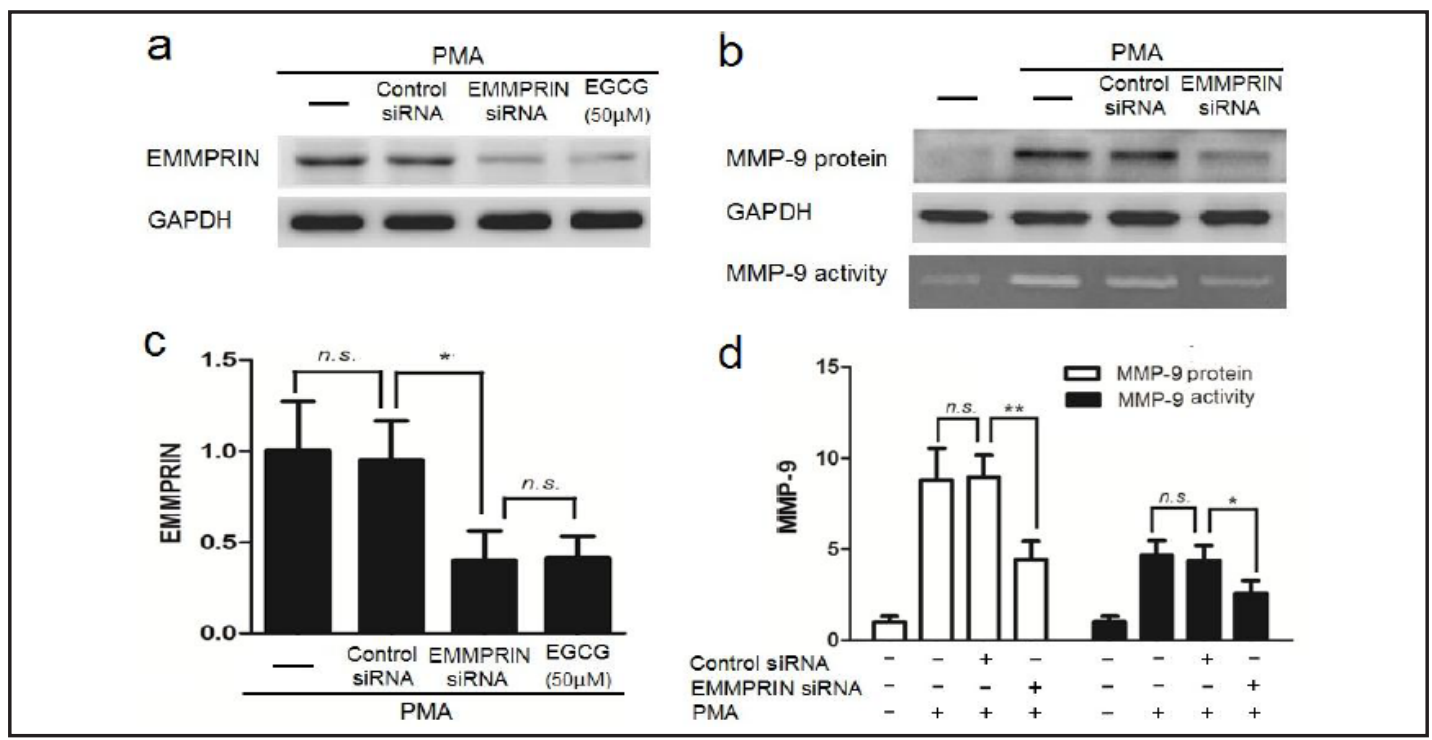

Fig. 3. Involvement of CD147 in EGCG-mediated inhibition of MMP-9 expression. THP-1 cells were transfected with EMMPRIN siRNA or scrambled siRNA prior to treatment with PMA. (a) Representative Western blot for EMMPRIN. As positive control, THP-1 cells were pretreated with EGCG (50 $\mu \mathrm{M})$ before exposure to PMA. (b) Representative Western blot for MMP-9 and representative gelatin zymography showing MMP-9 activity. (c) Densitometric analysis of EMMPRIN protein. (d) Densitometric analysis of MMP-9 protein and activity. Data represent mean \pm S.D. of three independent experiments. *: $\mathrm{P}<0.05$ vs control siRNA group, $* *$ : $\mathrm{P}<0.01$ vs control siRNA group, n.s.: $\mathrm{P}>0.05$.

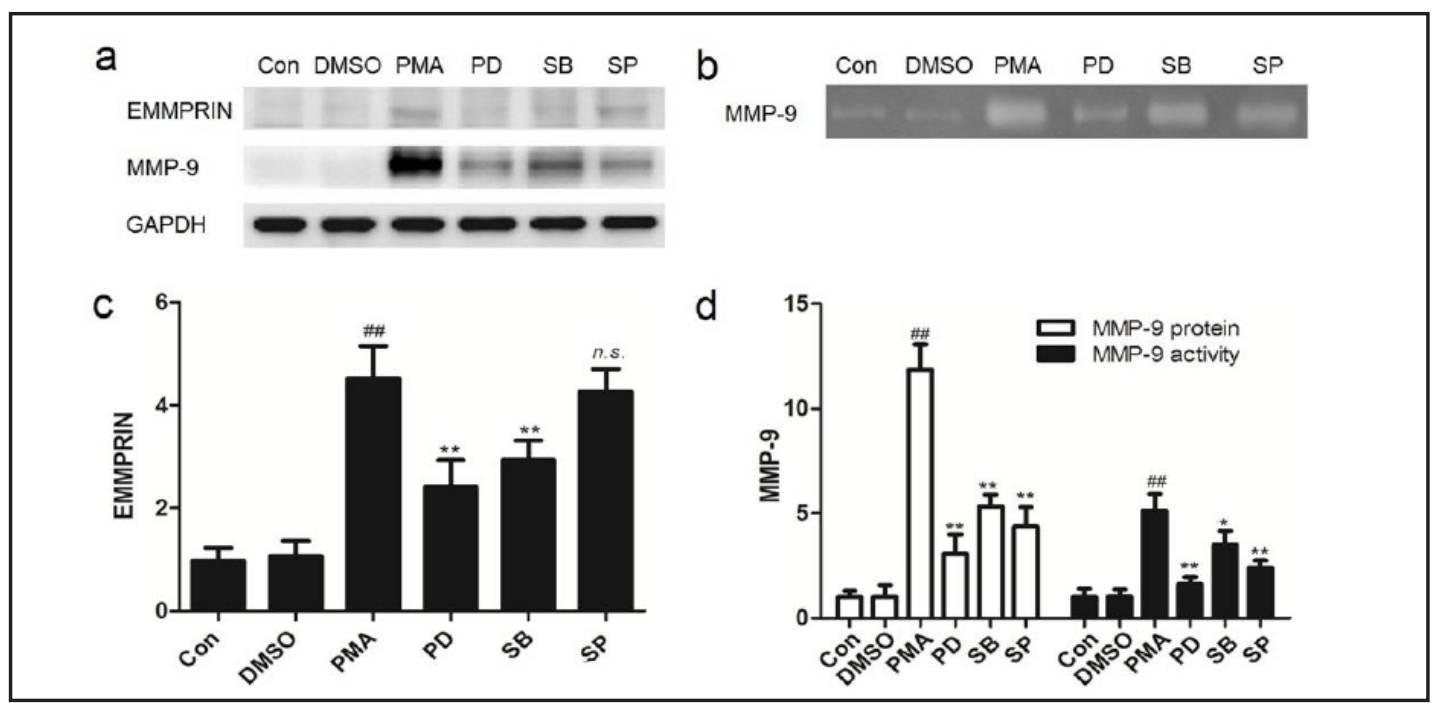

Fig. 4. Effects of MAPK inhibitors (PD98059, SB203580, and SP600125) on EMMPRIN and MMP-9 expression in PMA-induced macrophages. THP-1 Cells were pre-incubated with PD98059 (PD, $20 \mu$ M), SB203580 (SB, $10 \mu \mathrm{M}$ ), and SP600125 (SP, $10 \mu \mathrm{M}$ ) for 1 hour and then cultured with $100 \mathrm{nM}$ PMA for 48 hours. (a) Representative Western blots for EMMPRIN and MMP-9 detection. (b) Representative gelatin zymography showing MMP-9 activity. (c) Densitometric analysis of EMMPRIN protein. (d) Densitometric analysis of MMP-9 protein and activity. Data represent mean \pm S.D. of three independent experiments. \#\#: $\mathrm{P}<0.01$ vs control (con) group, *: P < 0.05 vs PMA group, **: P < 0.01 vs PMA group. n.s.: P > 0.05 vs PMA group.

JNK-specific inhibitor) on the expression of EMMPRIN and MMP-9 [29]. As shown in Fig. 4, the increased MMP-9 expression was abrogated by PD98059, SB203580 and SP600125. Moreover, PD98059 and SB203580 significantly suppressed EMMPRIN expression, whereas SP600125 had no similar effect. These results indicate that ERK1/2, p38 and JNK pathways 

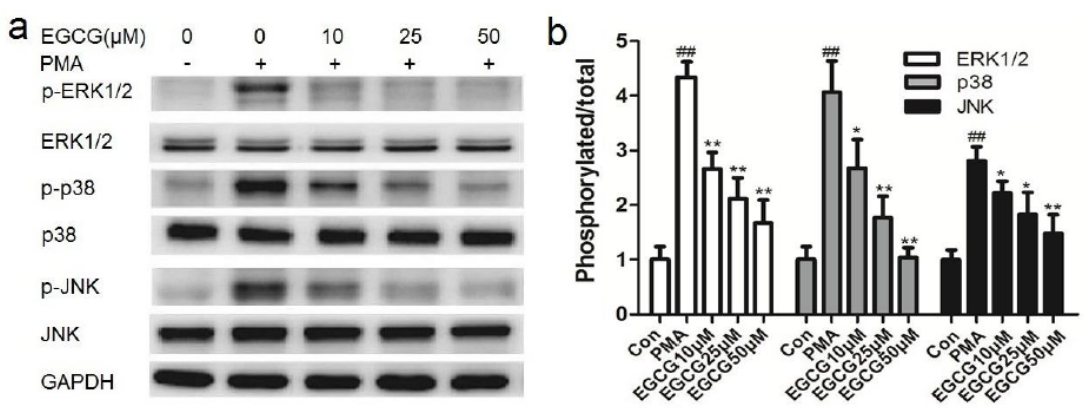

Fig. 5. EGCG inhibits the activation of ERK1/2, p38, and JNK pathways in PMA-induced THP-1 cells. THP-1 cells were pretreated with EGCG at increasing concentration $(10-50 \mu \mathrm{M})$ for 1 hour, followed by PMA for 5 minutes. (a) Western blot analysis was carried out using specific anti-bodies to phospho-ERK1/2, ERK1/2, phospho-p38, p38, phospho-JNK, JNK and GAPDH. (b) Quantification of the Western blot data was carried out by densitometric analysis using Image Lab software, and the ratio of phosphorylated protein/total protein was shown. The expression of GAPDH was used for protein level normalization. Data represent mean \pm S.D. of three independent experiments. \#\#: P < 0.01 vs control (con) group, *: P < 0.05 vs PMA group, **: P $<0.01$ vs PMA group.

Fig. 6. EGCG suppresses the expression of MMP-9 and EMMPRIN through 67LR. (a) Representative Western blots for 67LR detection in PMA-induced macrophages. Cells were treated with or without EGCG $(50 \mu \mathrm{M})$ for 48 hours. (b) Representative Western blots for EMMPRIN and MMP-9 detection. For the blockage of 67LR, THP1 cells were pre-incubated with 67LR-blocking antibody $(20 \mu \mathrm{g} / \mathrm{ml})$ or control mouse IgM for 1 hour, then treated with EGCG $(50 \mu \mathrm{M})$ for 1 hour before exposure to PMA (100 nM) for 48 hours. (c) Representative gelatin zymography showing MMP-9 activity. (d) Real-time PCR results of EMMPRIN mRNA and densitometric analysis of EMMPRIN protein. (e) Re-

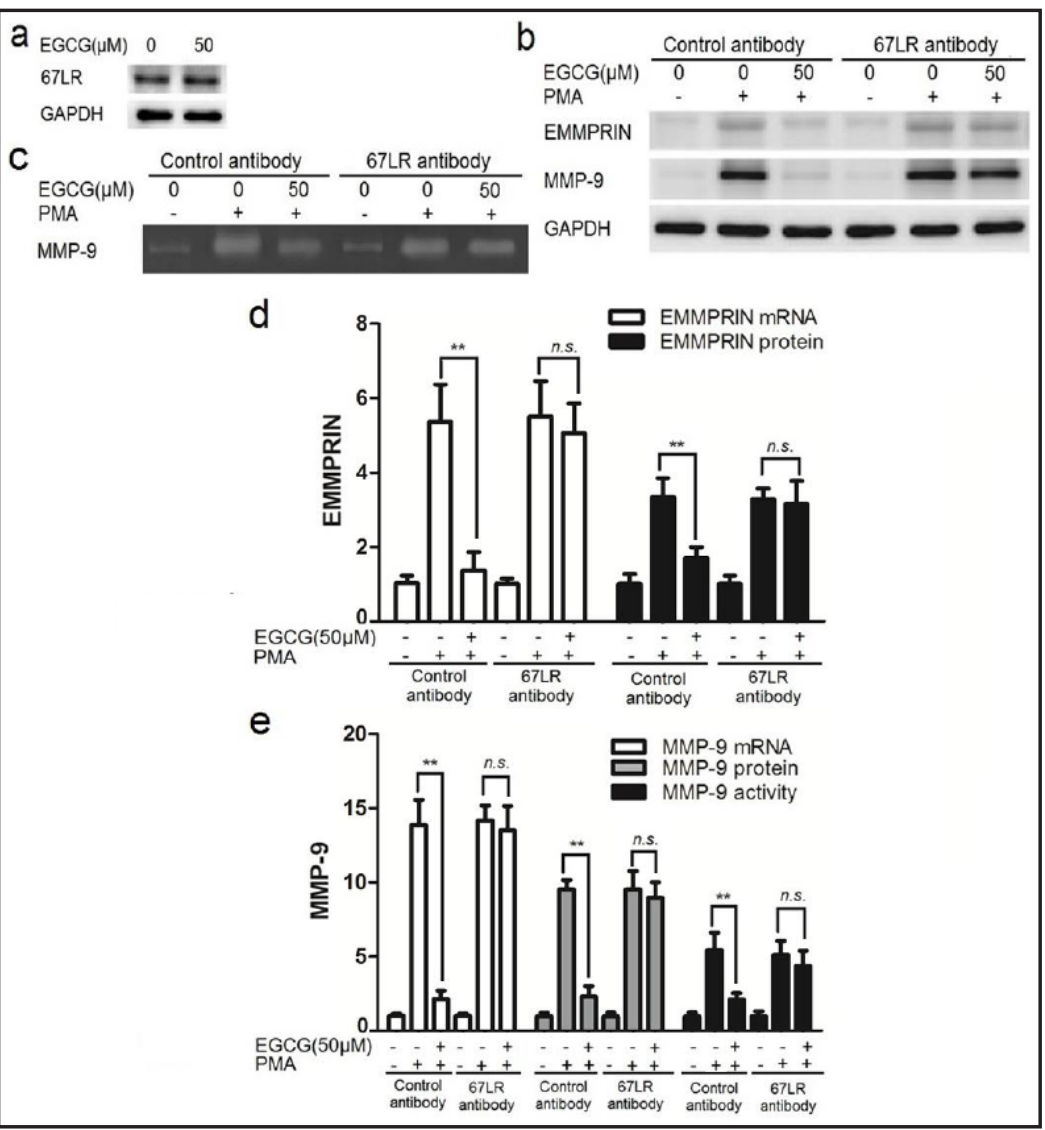
al-time PCR results of MMP-9 mRNA and densitometric analysis of MMP-9 protein and activity. Data represent mean \pm S.D. of at least three independent experiments. \#\#: $\mathrm{P}<0.01$ vs control (con) group, *: P 0.05 vs PMA group, **: $\mathrm{P}<0.01$ vs PMA group. n.s.: $\mathrm{P}>0.05$ vs PMA group.

are involved in the MMP-9 production stimulated by PMA, and ERK1/2 and p38 pathways are necessary for EMMPRIN expression. We then investigated the effects of EGCG on the activation of MAPK signaling pathways in PMA-induced THP-1 cells. The phosphorylation of ERK1/2, p38 and JNK was determined by Western blot using specific antibodies. THP-1 


\section{Cellular Physiology Cell Physiol Biochem 2016;39:2308-2319 \begin{tabular}{l|l|l} 
and Biochemistry $10.1159 / 000447923$ & (c) 2016 The Author(s). Published by S. Karger AG, Basel \\
wuww.karger.com/cpb
\end{tabular}

Fig. 7. EGCG inhibits the MAPK signal pathways through 67LR in PMA-induced THP-1 cells. For the blockage of 67LR, THP-1 cells were pre-incubated with 67LR-blocking antibody $(20 \mu \mathrm{g} / \mathrm{ml})$ or control mouse IgM for 1 hour before the treatment of EGCG. (a) Western blot analysis was carried out using specific anti-bodies to phospho-ERK1/2, ERK1/2, phospho-p38, p38, phospho-JNK, JNK and GAPDH. (b) Quantification of the Western blot data was carried out by densitometric analysis using Image Lab software, and the ratio of phosphorylated protein/ total protein was shown. The expression of GAPDH was used for protein level normalization. Data represent mean \pm S.D. of three independent experiments. \#\#: $\mathrm{P}<0.01$ vs control (con) group, *: $\mathrm{P}<$ 0.05 vs PMA group, ${ }^{* *}$ : $\mathrm{P}<0.01$ vs PMA group. n.s.: $\mathrm{P}>0.05$ vs PMA group.

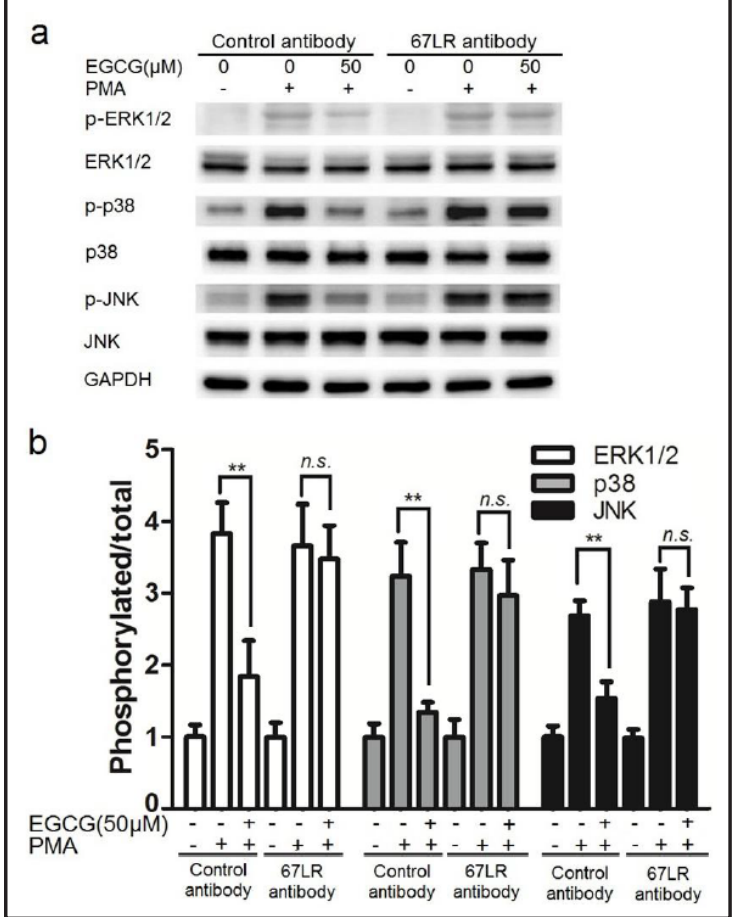

cells were pre-incubated with different concentrations of EGCG for 1 hour, and then treated with PMA for another 5 minutes [27, 30]. As shown in Fig. 5, EGCG significantly inhibited the activation of ERK1/2, p38, and JNK pathways.

67LR is involved in EGCG-mediated inhibition of EMMPRIN and MMP-9 expression

We evaluated the expression of 67LR in PMA-induced macrophages, the protein level of 67LR was not affected by treatment with $50 \mu \mathrm{M}$ EGCG for 48 hours (Fig. 6a). To explore whether 67LR could mediate the inhibitory effects of EGCG on EMMPRIN and MMP9 expression in PMA-induced macrophages, THP-1 cells were treated with neutralization antibody against 67LR $(20 \mu \mathrm{g} / \mathrm{ml})$ or control IgM for 1 hour prior to EGCG treatment. As shown in Fig. 6b-e, the blockage of 67LR resulted in abrogation of the inhibitory action of EGCG on EMMPRIN and MMP-9 expression. These results indicate that EGCG suppresses the expression of MMP-9 and EMMPRIN through 67LR.

67LR is involved in EGCG-mediated inactivation of the MAPK signaling pathways

Furthermore, we investigated whether EGCG suppressed PMA-induced MAPK signaling pathways activation through 67LR. THP-1 cells were pre-incubated with 67LR-blocking antibody or control IgM for 1 hour before EGCG treatment. As shown in Fig. 7, in anti-67LR antibody-treated cells, the inhibitory effects of EGCG on PMA-induced activation of MAPK signaling pathways were attenuated. Our results suggest that EGCG inhibits MAPK signaling pathways through 67LR.

\section{Discussion}

Atherosclerosis (AS) has been considered as a chronic inflammatory disease of the arterial wall. Monocytes/macrophages play an important role in all stages of atherosclerosis [31]. In the present study, we found that EGCG (10-50 $\mu \mathrm{mol} / \mathrm{L})$, the most important catechin present in green tea, significantly inhibited the expression of EMMPRIN and MMP-9 in a dose-dependent manner in PMA induced macrophages. Furthermore, these effects were mediated through 67LR and were via the inhibition of ERK1/2, p38, and JNK activation. 


\section{Cellular Physiology Cell Physiol Biochem 2016;39:2308-2319

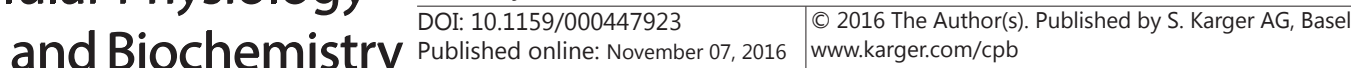

Wang et al.: Effects of EGCG on the Expression of EMMPRIN and MMP-9

Our previous studies have reported that consumption of green tea is associated with reduced risk of coronary artery disease [32,33]. Moreover, the antiatherosclerotic effect of green tea was also demonstrated in animal studies [34,35]. The underlying mechanisms for the protective effects of green tea include antioxidant, anti-inflammatory, antiproliferative, lipid-lowering and vasculoprotective properties of the green tea polyphenols $[17,36]$. However, it is not clear whether green tea polyphenol can promote stabilization of atherosclerotic plaque, which has been identified as a new therapeutic goal for preventing acute complications of atherosclerosis [37, 38]. Accumulating evidence now supports the concept that MMPs expression in monocytes and macrophages can promote atherosclerotic plaque rupture [6, 39]. Serum level of MMP-9 in ACS patients was significantly higher than that in patients with stable angina pectoris [40]. Fiotti et al. [41] found that the expression of MMP-9, but not TIMP-1 or MMP-2, was increased in plaques causing ACS. Overexpression of MMP-9 by macrophages in advanced atherosclerotic lesions induced acute plaque disruption in Apoe ${ }^{-/-}$mice [42]. Therefore, inhibitors of MMP-9 production from macrophages may be useful to prevent plaque rupture. Furthermore, MMP-9 deletion was proved to improve left ventricular function in remodeling myocardium post-MI [43]. In this study, we found that green tea polyphenol EGCG significantly inhibited the expression and activity of MMP-9 in a concentration-dependent manner in PMA-induced macrophages. More importantly, EGCG also inhibited the expression of EMMPRIN, which can induce the synthesis of MMPs and has recently been implicated in the development of atherosclerosis and atherothrombosis [14]. Moreover, downregulation of EMMPRIN significantly inhibited MMP-9 expression and enzymatic activity in PMA-induced macrophages, suggesting that EMMPRIN plays a key role in the inhibitory effects of EGCG on MMP-9 expression and activity. These findings indicate that EGCG is a potential therapeutic candidate for stabilizing atherosclerotic plaque.

Surface expression of 67LR has been found to be a dominant laminin-binding protein expressed in monocytes, neutrophils and macrophages [44]. Recently, this receptor has been identified as a cell-surface EGCG receptor that confers the anti-inflammatory action of EGCG. Several studies have reported that the inhibitory effects of EGCG on TLR2 and TLR4 signaling pathway are exerted through its binding to 67LR [22-24, 45]. Previously, we also found that EGCG inhibited TNF- $\alpha$-induced MCP-1 expression in HUVECs via suppression of NF- $\kappa B$ activation, and this effect was mediated by 67LR [25]. To our knowledge, no information is available concerning the 67LR-mediated effect of EGCG on MMP-9 and EMMPRIN expression. Here we showed, for the first time, that 67LR-blocking antibody blocked EGCG from the inhibitory effect on MMP-9 and EMMPRIN expression in PMA-induced macrophages, suggesting that EGCG may mediate this effect through 67LR. Taken together, these results indicate that 67LR may play an important role in the antiatherosclerotic and plaquestabilizing effects of EGCG.

As revealed in this study, PMA enhanced the phosphorylation of three major classes of MAPK signaling pathways, including ERK1/2, p38, and JNK. We found that the activation of ERK1/2, JNK and p38 pathways was involved in PMA-induced MMP-9 expression. Moreover, p38 and ERK pathways were involved in the upregulation of EMMPRIN expression, while JNK pathway was apparently uninvolved. These results agree well with the findings of Cao J et al. [28]. In previous studies, it has been demonstrated that 67LR, as a cell-surface EGCG receptor, mediates the inhibitory action of EGCG on the activation of MAPK pathways in LPS-induced dendritic cells [22] and endothelial cells [23]. Nonetheless, the relationship between 67LR and the inhibitory action of EGCG on MAPK pathways activation in PMAinduced human THP-1 macrophages remains unclear. Our data then showed that EGCG significantly inhibited PMA-induced phosphorylation of ERK1/2, p38 and JNK, suggesting that EGCG inhibits EMMPRIN and MMP-9 expression by regulating MAPK pathways in PMAinduced macrophages. Additionally, we found that anti-67LR antibody treatment resulted in abrogation of the inhibitory action of EGCG on PMA-induced activation of ERK1/2, p38, and JNK pathways. Our findings suggest that 67LR is responsible for mediating the inhibitory effect of EGCG on MMP-9 and EMMPRIN expression in PMA-induced macrophages and participates critically in the cell-surface interaction and MAPK signaling pathways.

\section{KARGER}




\section{Cellular Physiology Cell Physiol Biochem 2016;39:2308-2319 \begin{tabular}{l|l|l|} 
and BOI: 10.1159/000447923 & $\begin{array}{l}\text { C) 2016 The Author(s). Published by S. Karger AG, Basel } \\
\text { www.karger.com/cpb }\end{array}$
\end{tabular} \\ Wang et al.: Effects of EGCG on the Expression of EMMPRIN and MMP-9}

In conclusion, we show that green tea polyphenol EGCG inhibit the upregulation of EMMPRIN and MMP-9 expression in PMA-induced macrophages through 67LR. Our study reveals a novel mechanism underlying the downregulation of EMMPRIN and MMP-9 by EGCG. Considering the important role of EMMPRIN and MMP-9 in atherosclerotic plaque stability, our findings provide a new insight into the pharmacological role of EGCG in stabilizing atherosclerotic plaque. Further in vivo studies are needed to fortify our preliminary inference about the relationship between EGCG and stabilization of atherosclerotic plaque.

\section{Acknowledgments}

This work was supported by grants from the National Natural Science Foundation of China (No 81570363), the Natural ScienceYouth Foundation of the Jiangsu Province of China (No. BK20151034), and a project funded by the Priority Academic Program Development of Jiangsu Higher Education Institutions (PAPD, No. KYZZ15_0263).

\section{Disclosure Statement}

The authors declare no competing financial interests.

\section{References}

1 Falk E, Nakano M, Bentzon JF, Finn AV, Virmani R: Update on acute coronary syndromes: the pathologists' view. Eur Heart J 2013; 34: 719-728.

2 Ketelhuth DF, Bäck M: The role of matrix metalloproteinases in atherothrombosis. Curr Atheroscler Rep 2011;13:162-169.

3 Galis ZS, Khatri JJ: Matrix metalloproteinases in vascular remodeling and atherogenesis: the good, the bad, and the ugly. Circ Res 2002;90:251-262.

4 Yang YH, Li DL, Bi XY, Sun L, Yu XJ, Fang HL, Miao Y, Zhao M, He X, Liu JJ, Zang WJ: Acetylcholine Inhibits LPS-Induced MMP-9 Production and Cell Migration via the $\alpha 7$ nAChR-JAK2/STAT3 Pathway in RAW264.7 Cells. Cell Physiol Biochem 2015;36:2025-2038.

5 Schmidt R, Bultmann A, Ungerer M, Joghetaei N, Bulbul O, Thieme S, Chavakis T, Toole BP, Gawaz M, Schomig A, May AE: Extracellular matrix metalloproteinase inducer regulates matrix metalloproteinase activity in cardiovascular cells: implications in acute myocardial infarction. Circulation 2006;113:834-841. Newby AC: Metalloproteinase expression in monocytes and macrophages and its relationship to atherosclerotic plaque instability. Arterioscler Thromb Vasc Biol 2008;28:2108-2014.

7 Biswas C: Tumor cell stimulation of collagenase production by fibroblasts. Biochem Biophys Res Commun 1982;109:1026-1034.

8 Biswas C, Zhang Y, DeCastro R, Guo H, Nakamura T, Kataoka H, Nabeshima K: The human tumor cellderived collagenase stimulatory factor (renamed EMMPRIN) is a member of the immunoglobulin superfamily. Cancer Res 1995;55:434-439.

9 Iacono KT, Brown AL, Greene MI, Saouaf SJ: CD147 immunoglobulin superfamily receptor function and role in pathology. Exp Mol Pathol 2007;83:283-295.

10 Kim JY, Kim WJ, Kim H, Suk K, Lee WH: The Stimulation of CD147 Induces MMP-9 Expression through ERK and NF-kappaB in Macrophages: Implication for Atherosclerosis. Immune Netw 2009;9:90-97.

11 Hasaneen NA, Zucker S, Cao J, Chiarelli C, Panettieri RA, Foda HD: Cyclic mechanical strain-induced proliferation and migration of human airway smooth muscle cells: role of EMMPRIN and MMPs. FASEB J 2005;19:1507-1509.

12 Damsker JM, Okwumabua I, Pushkarsky T, Arora K, Bukrinsky MI, Constant SL: Targeting the chemotactic function of CD147 reduces collagen-induced arthritis. Immunology 2009;126:55-62. 


\section{Cellular Physiology Cell Physiol Biochem 2016;39:2308-2319 \begin{tabular}{l|l|l} 
and Biochemistry 10.1159/000447923 & (c) 2016 The Author(s). Published by S. Karger AG, Basel \\
wublished online: November 07,2016
\end{tabular} \\ Wang et al.: Effects of EGCG on the Expression of EMMPRIN and MMP-9}

13 Shackel NA, McGuinness PH, Abbott CA, Gorrell MD, McCaughan GW: Insights into the pathobiology of hepatitis $\mathrm{C}$ virus-associated cirrhosis: analysis of intrahepatic differential gene expression. Am J Pathol 2002;160:641-644.

14 Joghetaei N, Stein A, Byrne RA, Schulz C, King L, May AE, Schmidt R: The Extracellular Matrix Metalloproteinase Inducer (EMMPRIN, CD147) - a potential novel target in atherothrombosis prevention? Thromb Res 2013;131:474-480.

15 Yoon YW, Kwon HM, Hwang KC, Choi EY, Hong BK, Kim D, Kim HS, Cho SH, Song KS, Sangiorgi G: Upstream regulation of matrix metalloproteinase by EMMPRIN; extracellular matrix metalloproteinase inducer in advanced atherosclerotic plaque. Atherosclerosis 2005;180:37-44.

16 Xu S, Tang L, Mi Y, Jiang J, Zhu M, Chen B, Wang B, Li T, Xu C, Wang J, Lu X, Ge W, Wang L, Huang J: Clinical significance of leukotriene $\mathrm{b} 4$ and extracellular matrix metalloproteinase inducer in acute coronary syndrome. Clin Invest Med 2013;36:E282-289.

17 Babu PV, Liu D: Green tea catechins and cardiovascular health: an update. Curr Med Chem 2008;15:18401850.

18 Zhang YF, Xu Q, Lu J, Wang P, Zhang HW, Zhou L, Ma XQ, Zhou YH: Tea consumption and the incidence of cancer: a systematic review and meta-analysis of prospective observational studies. Eur J Cancer Prev 2015;24:353-362.

19 Tachibana H, Koga K, Fujimura Y, Yamada K: A receptor for green tea polyphenol EGCG. Nat Struct Mol Biol 2004;11:380-381.

20 Fujimura Y, Sumida M, Sugihara K, Tsukamoto S, Yamada K, Tachibana H: Green tea polyphenol EGCG sensing motif on the 67-kDa laminin receptor. PLoS One 2012;7:e37942.

21 Tsukamoto S, Hirotsu K, Kumazoe M, Goto Y, Sugihara K, Suda T, Tsurudome Y, Suzuki T, Yamashita S, Kim Y, Huang Y, Yamada K, Tachibana H: Green tea polyphenol EGCG induces lipid-raft clustering and apoptotic cell death by activating protein kinase $C \delta$ and acid sphingomyelinase through a $67 \mathrm{kDa}$ laminin receptor in multiple myeloma cells. Biochem J 2012;443:525-534.

22 Byun EB, Choi HG, Sung NY, Byun EH: Green tea polyphenol epigallocatechin-3-gallate inhibits TLR4 signaling through the 67-kDa laminin receptor on lipopolysaccharide-stimulated dendritic cells. Biochem Biophys Res Commun 2012;426:480-485.

23 Byun EB, Mi-SoYang, Kim JH, Song DS, Lee BS, Park JN, Park SH, Park C, Jung PM, Sung NY, Byun EH: Epigallocatechin-3-gallate-mediated Tollip induction through the 67-kDa laminin receptor negatively regulating TLR4 signaling in endothelial cells. Immunobiology 2014;219:866-872.

24 Bao S, Cao Y, Zhou H, Sun X, Shan Z, Teng W: Epigallocatechin gallate (EGCG) suppresses lipopolysaccharide-induced Toll-like receptor 4 (TLR4) activity via $67 \mathrm{kDa}$ laminin receptor (67LR) in 3T3-L1 adipocytes. J Agric Food Chem 2015;63:2811-2819.

25 Wang ZM, Gao W, Wang H, Zhao D, Nie ZL, Shi JQ Zhao S, Lu X, Wang LS, Yang ZJ: Green tea polyphenol epigallocatechin-3-gallate inhibits TNF- $\alpha$-induced production of monocyte chemoattractant protein-1 in human umbilical vein endothelial cells. Cell Physiol Biochem 2014;33:1349-1358.

26 Tsuchiya S, Kobayashi Y, Goto Y, Okumura H, Nakae S, Konno T, Tada K: Induction of maturation in cultured human monocytic leukemia cells by a phorbol diester. Cancer Res 1982;42:1530-1536.

27 Huang Z, Wang C, Wei L, Wang J, Fan Y, Wang L, Wang Y, Chen T: Resveratrol inhibits EMMPRIN expression via P38 and ERK1/2 pathways in PMA-induced THP-1 cells. Biochem Biophys Res Commun 2008;374:517521.

28 Cao J, Han Z, Tian L, Chen K, Fan Y, Ye B, Huang W, Wang C, Huang Z: Curcumin inhibits EMMPRIN and MMP-9 expression through AMPK-MAPK and PKC signaling in PMA induced macrophages. J Transl Med 2014;12:266.

29 Ahmad R, Shihab PK, Jasem S, Behbehani K. FSL-1 induces MMP-9 production through TLR-2 and NF- $\kappa$ B / AP-1 signaling pathways in monocytic THP-1 cells. Cell Physiol Biochem 2014;3:929-942.

30 Carter AB, Tephly LA, Hunninghake GW: The absence of activator protein 1-dependent gene expression in THP-1 macrophages stimulated with phorbol esters is due to lack of p38 mitogen-activated protein kinase activation. J Biol Chem 2001;276:33826-33832.

31 Cochain C, Zernecke A: Macrophages and immune cells in atherosclerosis: recent advances and novel concepts. Basic Res Cardiol 2015;110: 34.

32 Wang QM, Gong QY, Yan JJ, Zhu J, Tang JJ, Wang MW, Yang ZJ, Wang LS: Association between green tea intake and coronary artery disease in a Chinese population. Circ J 2010;74:294-300. 


\section{Cellular Physiology Cell Physiol Biochem 2016;39:2308-2319 \begin{tabular}{l|l|l} 
DOI: 10.1159/000447923 & $\begin{array}{l}\text { O 2016 The Author(s). Published by S. Karger AG, Basel } \\
\text { www.karger.com/cpb }\end{array}$
\end{tabular} \\ Wang et al.: Effects of EGCG on the Expression of EMMPRIN and MMP-9}

33 Wang ZM, Zhou B, Wang YS, Gong QY, Wang QM, Yan JJ, Gao W, Wang LS: Black and green tea consumption and the risk of coronary artery disease: a meta-analysis. Am J Clin Nutr 2011;93:506-515.

34 Chyu KY, Babbidge SM, Zhao X, Dandillaya R, Rietveld AG, Yano J, Dimayuga P, Cercek B, ShahPK: Differential effects of green tea-derived catechin on developing versus established atherosclerosis in apolipoprotein E-null mice. Circulation 2004;109:2448-2453.

35 Minatti J, Wazlawik E, Hort MA, Zaleski FL, Ribeiro-do-Valle RM, Maraschin M, da Silva EL: Green tea extract reverses endothelial dysfunction and reduces atherosclerosis progression in homozygous knockout lowdensity lipoprotein receptor mice. Nutr Res 2012;32:684-693.

36 Grassi D, Desideri G, Di Giosia P, De Feo M, Fellini E, Cheli P, Ferri L, Ferri C: Tea, flavonoids, and cardiovascular health: endothelial protection. Am J Clin Nutr 2013;98:1660S-1666S.

37 Libby P, Aikawa M: Stabilization of atherosclerotic plaques: new mechanisms and clinical targets. Nat Med 2002;8:1257-1262.

38 Nicholls SJ, Kataoka Y: Update in therapeutic approaches to plaque stabilization. Curr Atheroscler Rep 2014;16:392.

39 Bäck M, Ketelhuth DF, Agewall S: Matrix metalloproteinases in atherothrombosis. Prog Cardiovasc Dis 2010;52:410-428.

40 Fukuda D, Shimada K, Tanaka A, Kusuyama T, Yamashita H, Ehara S, Nakamura Y, Kawarabayashi T, Iida H, Yoshiyama M, Yoshikawa J: Comparison of levels of serum matrix metalloproteinase-9 in patients with acute myocardial infarction versus unstable angina pectoris versus stable angina pectoris. Am J Cardiol 2006;97:175-180.

41 Fiotti N, Altamura N, Orlando C, Simi L, Reimers B, Pascotto P, Zingone B, Pascotto A, Serio M, Guarnieri G, Giansante C: Metalloproteinases-2, -9 and TIMP-1 expression in stable and unstable coronary plaques undergoing PCI. Int J Cardiol 2008;127:350-357.

42 Gough PJ, Gomez IG, Wille PT, Raines EW: Macrophage expression of active MMP-9 induces acute plaque disruption in apoE-deficient mice. J Clin Invest 2006;116:59-69.

43 Jiang Y, Bai J, Tang L, Zhang P, Pu J. Anti-CCL21 antibody attenuates infarct size and improves cardiac remodeling after myocardial infarction. Cell Physiol Biochem 2015;37:979-990.

44 Tachibana H: Green tea polyphenol sensing. Proc Jpn Acad Ser B Phys Biol Sci 2011;87:66-80.

45 Byun EH, Omura T, Yamada K, Tachibana H: Green tea polyphenol epigallocatechin-3-gallate inhibits TLR2 signaling induced by peptidoglycan through the polyphenol sensing molecule 67-kDa laminin receptor. FEBS Lett 2011;585:814-820. 